\title{
Two approximate solutions to the Art Gallery Problem
}

\author{
Sanjay Rana \\ Centre for Advanced Spatial Analysis, University College London
}

\section{The Art Gallery Problem}

The Art Gallery Problem [Chvátal 1975] is a well known NP-hard computational geometry question, where the aim is to find the minimum number of observers required for complete visual coverage of an art gallery. Most existing solutions are based on heuristics that are dependent on the geometry and topology of the gallery i.e. whether the gallery is convex, rectilinear etc.

\section{Solutions based on discretisation of open space in the gallery}

In this proposal, a dense mesh of potential observers is placed in the open space that surrounds/immerses the obstacles in the gallery (Figure 1a) which basically discretises the open space. This aspect highlights the central assumption in this approach that the open space of the art gallery is covered with dense arrangement of observers. Each observer $v_{i}$ has a visual connectivity expressed as a set called isovist $a_{i}$ where $a_{i}=\left\{v_{i}, v_{j}, \ldots, v_{n}\right\} ; 1 \geq i, j, \ldots, n \leq \mathrm{N}, v_{j}, \ldots, v_{n}$ are observers visible from $v_{i}$ and $N$ is the set of all observers in the gallery. $\left|a_{i}\right|$ is referred as the rank of the observer. With this premise, the following algorithms are proposed to solve the art gallery problem.

\subsection{Rank and Overlap Elimination (ROPE)}

Originally developed for the extraction of the structure of architectural open spaces, ROPE [Rana and Batty, 2004] is essentially a greedy-search method. The ROPE method starts with the selection of the highest ranking observer and removal of lower ranking observers present in the isovist of high ranking observer. This step is then repeated with the next remaining highest ranking observer and continued so till a set of observers with a minimal overlap of isovists in the open space is achieved. The following pseudo-code shows how ROPE can be used to solve the art gallery problem:

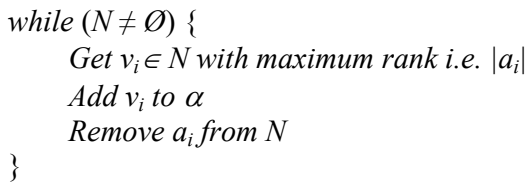

where $\alpha$ is the set of optimal observers.

Figure $1 \mathrm{~b}$ shows the output of the ROPE method revealing the location of 3 optimal observers. While for many gallery shapes the ROPE method will successfully yield the location and number of optimal observers, in certain shapes it will produce a reduced set of observers which although guaranteeing complete visual coverage of the gallery may not necessarily be the optimal observers. The gallery in Figure 1a is such an exceptional shape. As seen in Figure 1b the ROPE method suggests 3 optimal observers, however
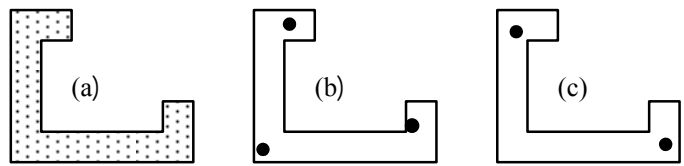

Figure 1. (a) An Art Gallery with a dense mesh of potential observers and the locations of optimal observers based on (b) ROPE and (c) CSC methods.

only 2 are needed. These types of shapes can be solved using the following novel brute-force heuristics.

\subsection{Combinatorial Set Coverage (CSC)}

The CSC method is essentially an iterative search method that looks for a combination of observers, whose ranks add up to the total number of observers in the open space. For example, if an observer $v_{i}$ satisfies the following condition:

$$
|N|=\left|a_{i}\right|
$$

then $v_{i}$ is the optimal observer to cover the entire gallery. If no single optimal observer is found then a search for two optimal observers can be similarly based on the condition,

$$
|N|=\left|a_{i} \cup a_{j}\right|
$$

The search can be extended further up to the upper limit of the optimal observers till a solution is found. The upper limit of the number of optimal observers can be found using the ROPE method or formulas such as by Chvátal [1975]. Figure 1c shows the location of the two optimal observers in the gallery. One of the unique aspects of the CSC method is that it converts the otherwise complex spatial problem into one of trivial set union and inequality tests.

\section{Conclusion}

Admittedly, the ROPE and CSC methods do not solve the NP-hard aspect of the art gallery problem, but they do suggest that the art gallery problem is NP-hard only in worst cases. The proposed algorithm will be particularly useful in real practical applications. In particular, they can be applied for 3D spaces and arbitrary topological arrangements (e.g. non-convex polygons, polygons with holes, rectilinear galleries, and line segments) without any modification to the algorithms. Both ROPE and CSC methods would terminate in polynomial (or even better e.g. $O(n-l)$ in the case of one optimal observer) time in the worst cases.

\section{References}

CHVÁTAL, V. 1975. A Combinatorial Theorem in Plane Geometry. Journal of Combinatorial Theory 18, 39-41.

RANA, S. AND BATTY, M. 2004. Visualising the structure of Architectural Open Spaces based on Shape Analysis. International Journal of Architectural Computing 2, 1, 123 -132. 